\title{
ON SATURATED FORMATIONS WHICH ARE SPECIAL RELATIVE TO THE STRONG COVERING-AVOIDANCE PROPERTY
}

\author{
J. C. BEIDLEMAN AND A. R. MAKAN
}

ABSTRACT. Let $\mathcal{F}$ be a saturated formation of finite soluble groups. Let $G$ be a finite soluble group and $F$ an $F$-projector of $G$. Then $F$ is said to satisfy the strong covering-avoidance property if (i) $F$ either covers or avoids each chief factor of $G$, and (ii) $F \cap L / F \cap K$ is a chief factor of $F$ whenever $L / K$ is a chief factor of $G$ covered by $F$. Let $C_{\mathcal{F}}$ denote the class of all finite soluble $G$ in which the $\mathcal{F}$-projectors satisfy the strong covering-avoidance property. $\mathcal{C}_{\mathcal{F}}$ is a formation. Let $\mathscr{y}_{\mathcal{F}}$ be the class of groups $G$ in which an $\mathcal{F}$-normalizer is also an $\mathcal{F}$-projector. $y_{\mathcal{F}}$ is a formation studied by Klaus Doerk. Note that $\mathscr{y}_{\mathcal{F}} \subseteq \mathcal{C}_{\mathcal{F}}$. $\mathcal{F}$ is said to be $\mathcal{C}$-special if $\mathcal{C}_{\mathcal{G}}=\mathscr{Y}_{\mathrm{GF}}$. The purpose of this note is to study $\mathcal{C}$-special formations. Two characterizations of $\mathcal{C}$-special formations are given. Let $i$ be a positive integer and let $\pi^{(i)}$ denote the class of finite soluble groups $G$ whose $F$ itting length is at most $i$. Then $r^{(i)}$ is $\mathcal{C}$-special. Finally, the formation $\mathcal{C}_{\mathcal{F}}$ is saturated if and only if $\mathcal{F}$ is the class of all finite soluble groups.

Let $\mathcal{F}$ be a saturated formation which contains the class $\pi$ of finite nilpotent groups as a subformation and let $\mathcal{P}$ be a property which a subgroup of a finite group may possess. The class of all finite soluble groups in which the $\mathcal{F}$-projectors have property $\mathcal{P}$ is denoted by $\mathcal{P}_{\mathcal{F}} \cdot \mathcal{F}$ is said to be special relative to $\mathcal{P}$ or simply $\mathcal{P}$-special if $\mathscr{P}_{\text {ff }}$ coincides with the formation $\mathcal{Y}_{\mathcal{F}}$ of all finite soluble groups in which the $\mathcal{F}_{\text {-normalizers are also the }}$ $\mathcal{F}$-projectors.

Let $\mathcal{P}$ be defined as follows: A subgroup $H$ of $G$ is said to have property $\mathcal{P}$ if $G=C_{G}(R / S) H$ whenever $R / S$ is a chief factor of $G$ covered by $H$. One can easily check that in this case $\mathcal{P}_{\mathcal{F}}=\mathscr{B}_{\mathcal{F}}$, the class of all finite soluble groups $G$ in which the $\mathcal{F}$-projectors cover only the $\mathcal{F}$-central chief factors of $G$ (see Doerk [2, Definition 4.1 (a)]). In [2], Doerk characterizes

Received by the editors September 4, 1973.

AMS (MOS) subject classifications (1970). Primary 20D10.

Key words and phrases. Saturated formation, soluble, projector, normalizer. 
those saturated formations which are special relative to this property $\mathcal{P}$. In particular, he shows (cf. [2, Satz 4.11]) that $\mathcal{F}$ is $\mathcal{P}$-special if and only if $\mathcal{F}=s_{p}, \mathcal{F}(p)$ for some prime $p$, where $\{\mathcal{F}(q)\}$ is the canonical definition of $\mathcal{F}$ and $S_{p}$, is the class of all finite soluble $p^{\prime}$-groups. He also characterizes (cf. [2, Satz 4.9]) those saturated formations $\mathcal{F}$ for which $\mathscr{P}_{\mathcal{F}}=\mathfrak{B}_{\mathcal{F}}$.

The aim of this paper is to investigate the $\mathcal{C}$-special saturated formations, where $\mathcal{C}$ denotes the strong covering-avoidance property. A subgroup $H$ of a finite group $G$ is said to have the strong covering-avoidance property if (i) $H$ either covers or avoids each chief factor of $G$, and (ii) $H \cap L / K \cap L$ is a chief factor of $H$ whenever $L / K$ is a chief factor of $G$ covered by $H$. In view of Theorem 4.1 of Carter and Hawkes [1], an $\mathcal{F}$-normalizer of any finite soluble group has the strong covering-avoidance property. Hence, $\mathcal{Y}_{\mathcal{F}}$ is always contained in $\mathcal{C}_{\boldsymbol{f}}$.

In $\S 2$, we discuss a simple characterization and give some examples of saturated formations which are $\mathcal{C}$-special.

In $\$ 3$, we show that, unlike the case of the property $\mathcal{P}$ defined above, the only $\mathcal{C}_{\text {-special saturated formations }} \mathcal{f} \neq\{1\}$ for which $\mathcal{C}_{\mathcal{F}}$ is saturated is the class of all finite soluble groups.

Throughout this paper only finite soluble groups are considered. $\mathcal{F}$ will always denote a saturated formation containing $\pi$. Further, $\{\mathcal{F}(p)\}$ will always denote the canonical definition of $\mathcal{F}$; that is for each prime $p, s, \mathcal{F}(p)=$ $\mathcal{F}(p) \subseteq \mathcal{F}$ and $\{\mathcal{F}(p)\}$ locally define $\mathcal{F}$. Also, for each prime $p, \mathcal{F}^{*}(p)$ denotes, as in Doerk [2], the class of all groups whose $\mathcal{F}$-projectors belong to $\mathcal{F}(p) . \mathcal{F}^{*}(p)$ is a formation. A $p$-chief factor $H / K$ of the group $G$ is called $\mathfrak{F}^{*}$-central if $G / C_{G}(H / K) \in \mathcal{F}^{*}(p)$. If $H / K$ is not $\mathcal{F}^{*}$-central, then it is termed $\mathcal{F}^{*}$-eccentric. The notation used is standard. For various basic definitions and terminology unexplained here we refer the readers to Carter and Hawkes [1], Gaschütz [4] and Huppert [6].

2. A characterization and some examples of $C$-special formations. We begin this section by showing

Proposition 1. $\mathcal{C}_{\mathcal{F}}$ is a formation.

For the proof of Proposition 1 we need the following.

Lemma 2. Let $G$ be a group and $V$ an F-projector of $G$. If $V$ covers or avoids each chief factor in a given chief series of $G$ and intersects the latter into a chief series of $V$, then $V$ has the strong covering-avoidance property. 
Proof. By induction on $|G|$. We first show that $V$ has the coveringavoidance property in $G$. Let $A$ be the minimal normal subgroup of $G$ in the given chief series of $G$, and let $H / K$ be an arbitrary chief factor of $G$. If $H / K$ is $\mathcal{F}^{*}$-central, then, by a result of Dade (cf. [2, Satz 2.9]), $V$ covers $H / K$. Hence, we can assume that $H / K$ is $\mathcal{F}^{*}$-eccentric. First, assume that $A$ avoids $H / K$. Then $A H: A K$ is an $\mathcal{F}^{*}$-eccentric chief factor of $G$, hence avoiding $V$. By induction $V A / A$ either covers or avoids a chief factor of $G / A$. By Huppert [7, Satz 2.1],

$$
V \cap H \subseteq V \cap A H \subseteq V \cap A K=(V \cap A)(V \cap K)
$$

so that,

$$
V \cap H=(V \cap K)(V \cap H \cap A) \subseteq K
$$

since $A$ avoids $H / K$. Thus, $V$ avoids $H / K$ in this case.

Suppose next that $A$ covers $H / K$. Then $A$ is $G$-isomorphic to $H / K$, and so, $A$ is $\mathfrak{F}^{*}$-eccentric. Hence, $V \cap A=1$. Because of Huppert $[7$, Satz 2.1], we have

$$
V \cap H=V \cap K A=(V \cap K)(V \cap A)=V \cap K
$$

and $V$ avoids $H / K$.

It remains to show that $V \cap H / N \cap K$ is a chief factor of $V$ whenever $H / K$ is a chief factor of $G$ covered by $V$. If $A$ avoids $H / K$, then $H A / K A$ is a chief factor of $G / A$ which is covered by $V A / A$, and so, by induction, $H A \cap V A / K A \cap V A$ is a chief factor of $V A$. But

$$
\begin{aligned}
H A \cap V A / K A \cap V A & =A(V \cap H) / A(V \cap K) \\
& \cong(H \cap V / A \cap V) /(K \cap V / A \cap V)
\end{aligned}
$$

whence $H \cap V / K \cap V$ is a chief factor of $V$. Hence, assume that $A$ covers $H / K$. Then $A$ is $G$-isomorphic to $H / K$, hence by $[2$, Satz 2.9] $V$ covers $A$. By assumption, $A$ is a chief factor of $V$, hence $H \cap V / K \cap V$ is a chief factor of $V$. This completes the proof.

Proof of Proposition 1. We show first that $\mathcal{C}_{\mathcal{F}}$ is a homomorph. Let $G \in \mathcal{C}_{\mathcal{F}}, N \unlhd G$ and let $F$ be an $\mathcal{F}$-projector of $G$. Let $(H / N) /(K / N)$ be a chief factor of $G / N$, then $H / K$ is a chief factor of $G$, and so, $F N / N$ either covers or avoids $(H / N) /(K / N)$. Assume that $F N / N$ covers $(H / N) /(K / N)$. Then $F$ covers $H / K$, hence $H \cap F / K \cap F$ is a chief factor of $F$. Thus,

$$
(H \cap F / N \cap F) /(K \cap F / N \cap F)
$$

is a chief factor of $F / N \cap F$ and so $(H \cap F N / N) / K \cap F N / N)$ is a chief factor of $F N / N$. Hence, $G / N \in \mathcal{C}_{\mathcal{G}}$. 
Next, we show: $G \in \mathcal{C}_{\mathcal{F}}$ whenever $G$ has two distinct minimal normal subgroups $N$ and $M$ such that both $G / N$ and $G M$ belong to $\mathcal{C}_{c F}$. Let $F$ be an $\mathcal{F}$-projector of $G$ and let $H / K$ be a chief factor of $G$ above $N$. Since $G / N \in \mathcal{C}_{\mathcal{F}}, F N / N$ either covers or avoids $(H / N) /(K / N)$, and if it covers it then $(H \cap F N / N) /(K \cap F N / N)$ is a chief factor of $F N / N$. Clearly, $F$ either covers or avoids $H / K$, and if it covers $H / K$, then since

$$
(H \cap F N / N) /(K \cap F N / N) \cong H \cap F / K \cap F,
$$

$H \cap F / K \cap F$ is a chief factor of $F$. Similarly, since $G / M \in \mathcal{C}_{\mathcal{F}}, F M / M$ either covers or avoids $N M / M$, and if it covers it then $N M / M$ is a chief factor of $F M / M$. If $F M / M$ avoids $N M M M$, then

$$
F \cap N \subseteq(F \cap N M) \cap N \subseteq M \cap N=1,
$$

and so $F$ avoids $N$. On the other hand, if $F M / M$ covers $N M / M$, then $N M \subseteq$ $F M$, and so, $N M=M(F \cap N M)=M(F \cap N)$ by Huppert [7, Satz 2.1]. An order argument now shows that $N=N \cap F$ and so $F$ covers $N$. Moreover, $N$ is a chief factor of $F$ since $N M / M$ is a chief factor of $F M / M$. The proposition now follows from Lemma 2.

We now give a simple characterization of $C$-special formations. For this purpose we introduce the following definition. The group $G$ is said to be $\mathcal{F}^{*}(p)$-irreducible if either $G=\{1\}$ or (i) $G \in \mathcal{F}^{*}(p) \cap \mathcal{C}_{\mathcal{G}}$ and (ii) $G$ has a faithful, irreducible $Z_{p}[G]$-module $V$ such that $V_{F}$, the restriction of $V$ to $F$, an $\mathcal{F}$-projector of $G$, is an irreducible $Z_{p}[F]$-module.

For each prime $p$, let $\alpha(p)$ denote the class of $\mathfrak{F}^{*}(p)$-irreducible groups and $A(p)$ the formation generated by $\alpha(p)$.

Remark. Let $p$ be a prime. Then $a(p) \subseteq \mathfrak{F}(p)$ if and only if $\mathcal{F}(p)=$ $S_{p} A(p)$.

Proof. Assume that $a(p) \subseteq \mathcal{F}(p)$ and suppose that $\mathcal{F}(p) \neq S_{p} A(p)$. Let $G \in \mathcal{F}(p) \backslash S_{p} A(p)$ be of minimal order. Then $G$ has a unique minimal normal subgroup $N$ such that $G / N \in S_{p} A(p)$. Hence, $N$ is an elementary abelian $q$-group, where $q$ is a prime distinct from $p$. By Doerk [2, Hilfssatz 1.3], $G$ has a faithful, irreducible $Z_{p}[G]$-module $V$, whence $G \in a(p) \subseteq S_{p} A(p)$, which is a contradiction. Hence, $\mathcal{F}(p)=S_{p} A(p)$.

The converse is immediate.

Theorem 3. $\mathfrak{F}$ is $\mathcal{C}$-special if and only if $\alpha(p) \subseteq \mathfrak{F}(p)$ for each prime $p$.

Proof. Assume that $\mathcal{F}$ is $\mathcal{C}$-special and let $\{1\} \neq G \in \alpha(p)$. Let $V$ be a faithful, irreducible $Z_{p}[G]$-module such that $V_{F}$, where $F$ is an $\mathcal{F}_{\text {-projector }}$ 
of $G$, is irreducible. Let $H=[V] G$ be the semidirect product of $V$ by $G$. Since $G \in \mathcal{F}^{*}(p)$, it follows by Doerk [2, Hilfssatz 2.6] that $V F$ is an $\mathcal{F}_{\text {-pro- }}$ jector of $H$. Further, since $G \in a(p), H \in \mathcal{C}_{\mathcal{f}}$. By our assumption, $\mathcal{C}_{\mathcal{f f}}=$ $\mathcal{Y}_{\mathcal{F}}$, so that $V$ is $\mathcal{F}$-central in $H$. Hence, $G \cong H / V \in \mathcal{F}(p)$ and so $\alpha(p) \subseteq$ $\mathcal{F}(p)$.

Conversely, assume that $\alpha(p) \subseteq \mathcal{F}(p)$ for each prime $p$. Further, assume that $\mathcal{C}_{\mathcal{F}} \neq \mathcal{Y}_{\mathcal{F}}$ and let $G \in \mathcal{C}_{\mathcal{F}} \backslash \mathcal{Y}_{\mathcal{F}}$ be of minimal order. Then $G$ has a unique minimal normal subgroup $N$ and $G / N \in \mathscr{Y}_{\mathcal{F F}}$. Since $G \notin \mathcal{Y}_{\mathcal{F F}}$, an $\mathcal{F}_{\text {-projector }}$ $F$ of $G$ covers $N$. Let $|N|=p^{n}, n>0$, and assume that $C_{G}(N) \neq N$. Let $L=G / C_{G}(N)$ and let $H=[N] L$, the semidirect product of $N$ by $L$. By Huppert [6, Hilfssatz 7.21, p. 70], $H \in \mathcal{C}_{\mathcal{G}}$. Since $|H|<|G|, H \in \mathcal{Y}_{\mathcal{F}}$. Hence $L \cong H / N \in \mathcal{F}(p)$, since $N$ is $\mathcal{F}^{*}$-central in $H$. But then $G \in \mathcal{Y}_{\mathcal{F}}$, a contradiction. Therefore, $N=C_{G}(N)$ and so $N$ is complemented in $G$ by $M$, say. Now, since $N$ is covered by $F$ and is a self-centralizing chief factor of $F$, it follows that $F \cap M \in \mathcal{F}(p)$. By [1, Theorem 5.12] we can assume that $F \cap M$ is an $\mathcal{F}$-projector of $M$. Hence, $M \in \alpha(p) \subseteq \mathcal{F}(p)$ so that $G \in S_{p} \mathcal{F}(p)=\mathscr{F}(p)$ $\subseteq \mathcal{F} \subseteq \mathcal{Y}_{\mathcal{F}}$, a contradiction. This completes the proof.

The saturated formation $\mathcal{F}$ is said to satisfy condition $C$ provided that if $G$ is a primitive group with unique minimal normal subgroup $N$, and $F$ is an $\mathcal{F}$-projector of $G$ with unique minimal normal subgroup $N$, then $F=G$.

Theorem 4. $\mathcal{F}$ is $\mathcal{C}$-special if and only if $\mathfrak{F}$ satisfies condition $C$.

Proof. Assume that $\mathcal{F}$ satisfies condition $C$ and let $\{1\} \neq G \in \alpha(p)$. Let $V$ be a faithful, irreducible $Z_{p}[G]$ module such that $V_{F}, F$ an $\mathcal{F}$-projector of $G$, is an irreducible $Z_{p}[F]$-module. Let $H=[V] G$ be the semidirect product of $V$ by $G$. By Doerk [2, Hilfssatz 2.6], $\bar{F}=V F$ is an $\mathcal{F}$-projector of $H$. Since $H$ is a primitive group and $V_{F}$ is irreducible, it follows that $G=F \epsilon$ $\mathcal{F}(p)$. Hence, $\mathcal{F}$ is $\mathcal{C}$-special by Theorem 3 .

Conversely, assume that $\mathcal{F}$ is $\mathcal{C}$-special. Let $G$ be a primitive group with unique minimal normal subgroup $N$ and let $M$ complement $N$ in $G$. Assume that the $\mathcal{F}$-projector $F$ of $G$ covers $N$ and let $|N|=p^{n}, n>0$. Be-

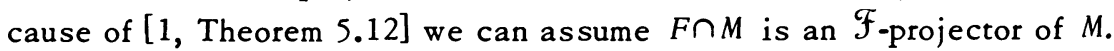
Suppose that $N$ is the unique minimal normal subgroup of $F$. Then $M \epsilon$ $\alpha(p) \subseteq \mathcal{F}(p)$ because of Theorem 3 , hence $G=[N] M \in S_{p} \mathcal{F}(p) \subseteq \mathcal{F}$. This completes the proof.

The saturated formation $\mathcal{F}$ is said to satisfy condition A (cf. Huppert $\left[7\right.$, p. 569]) if there exists a formation $X$ such that $\mathcal{F}(p)=s_{p} \mathcal{X}$ for each prime $p$. Further, $\mathcal{F}$ is said to satisfy condition B (cf. Huppert [7, p. 569]) 
if $\mathcal{F}$ is subgroup-inherited and if $G \in \mathcal{F}$ and $N$ is a minimal normal subgroup of $G$, then Aut $(N) \in \mathcal{F}$.

Proposition 5. If $\mathcal{F}$ satisfies either condition A or condition $\mathrm{B}$, then $\mathcal{F}$ is C-special.

Proof. Because of Theorem 4 it is enough to show that $\mathcal{F}$ satisfies condition $C$. Let $G$ be a primitive group with unique minimal normal subgroup $N$, and let $F$ be an $\mathcal{F}$-projector of $G$ which contains $N$ as its unique minimal normal subgroup.

Assume that $\mathcal{F}$ satisfies condition $B$. Then $G / N \in \mathcal{F}$, hence $G=F$ and $\mathcal{F}$ satisfies condition $C$.

Next assume that $\mathcal{F}$ satisfies condition $A$ and let $\mathcal{X}$ be a formation such that $\mathcal{F}(p)=S_{p} \mathcal{X}$ for each $p$. Assume that $G^{\mathfrak{F}} \neq\{1\}$. If $N=G^{\mathfrak{F}}$, then $G=F$, a contradiction. Hence, $N$ is a proper subgroup of $G^{\mathcal{F}}$. Let $N$ be a power of the prime $p$. Then

$$
G / G^{\mathfrak{F}} \cong F / F \cap G^{\mathfrak{F}} \cong(F / N) /\left(F \cap G^{\mathscr{F}} / N\right) \in X \subseteq \mathcal{F}(p) .
$$

Let $G / \mathcal{F}$ be a $q$-chief factor of $G$. Since $G^{\mathcal{F}} \subseteq C_{G}\left(G^{\mathcal{F}} / K\right)$, it follows that $G / C_{G}\left(G^{\mathcal{F}} / K\right) \in \mathcal{X}$ and so $G{ }^{\mathcal{F}} / K$ is an $\mathcal{F}$-central chief factor of $G$. This contradiction completes the proof.

Let $\gamma^{(0)}$ denote the class of groups consisting of the group of order 1 , and for each positive integer $i$, let $\Re^{(i)}$ denote the formation of all groups $G$ of Fitting length at most $i$. Then, for $i \geq 1, \Re^{(i)}$ has a canonical definition $\left\{S_{p} \Re^{(i-1)}\right\}$. Hence, the saturated formations $\Re^{(i)}, i \geq 1$, satisfy condition $A$ and so are $\mathcal{C}$-special. The saturated formation $\bar{X}$ of supersoluble groups satisfies condition $B$, hence $X$ is $\mathcal{C}$-special. Saturated formations $\mathcal{F}$ satisfying condition $B$ are easily classified. This will be the content of a later paper by the first author.

3. C-special formations for which $\mathcal{C}_{\xi}$ is saturated. In this section, we show

Theorem 6. $\mathcal{C}_{\mathcal{F}}$ is saturated if and only if $\mathcal{F}=S$, the class of all finite soluble groups.

In order to prove this theorem we need the following lemma.

Lemma 7. If $\mathcal{C}_{\mathcal{F}}$ is locally defined by the family $\left\{\mathcal{C}_{\mathcal{F}}(p)\right\}$, one for each prime $p$, of full and integrated homomorphs then $\mathcal{C}_{\mathcal{F}}=\Re \mathfrak{F}$. In particular, $\mathcal{F}$ is C-special. 
Proof. Since $\Re \mathcal{F} \subseteq \mathcal{C}_{\mathcal{F}}, s_{p} \mathcal{F} \subseteq \mathcal{C}_{\mathcal{F}}(p)$ for each prime $p$. Hence, it suf-

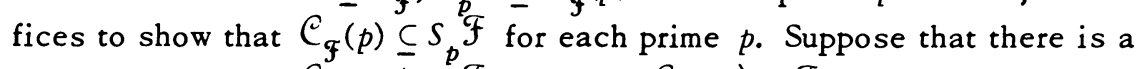
prime $p$ such that $\mathcal{C}_{\mathcal{F}}(p) \notin s_{p}^{p} \mathcal{F}$. Let $G \in \mathcal{C}_{\mathcal{F}}(p) \backslash S_{p} \mathcal{F}$ be of minimal order. Since $\mathcal{C}_{\boldsymbol{f}}(p)$ is a homomorph and $S_{p} \mathcal{F}$ is a saturated formation, $G$ has a unique minimal normal subgroup $N$ which is self-centralizing in $G$. Let $|N|=q^{n}, n>0$. Clearly $q \neq p$. Let $F$ be an $\mathcal{F}$-projector of $G$ and assume first that $F$ avoids $N$. Let $M$ be a complement of $N$ in $G$ which contains $F$. By Doerk [2, Hilfssatz 1.2], $G$ has a faithful, irreducible $Z_{p}[G]$-module $V$ such that $V_{M}$ contains the irreducible, trivial $Z_{p}[M]$-module as a factor module. In particular, $V_{F}$ is not an irreducible $Z_{p}[F]$-module. Let $H=$ $[V] G$. Since $G \in \mathcal{C}_{\mathcal{F}}(p) \subseteq \mathcal{C}_{\mathcal{F}}$, we have $H \in S_{p} \mathcal{C}_{\mathcal{F}}(p)=\mathcal{C}_{\mathcal{F}}(p) \subseteq \mathcal{C}_{\mathcal{F}}$. Thus, since $C_{V}\left(F^{\mathfrak{F}(p)}\right) \neq\{1\}, V F$ is an $\mathcal{F}$-projector of $H$. However, $V$ is not a chief factor of $V F$, though it is a chief factor of $H$, contrary to $H \in \mathcal{C}_{\mathfrak{f}}$.

Assume next that $F$ covers $N$. In particular, since $N$ is self-centralizing in $G, F \in \mathcal{F}(q)$. For the same reason, $G \in \mathcal{C}_{\mathcal{F}}(q)$ since $G \in \mathcal{C}_{\mathcal{F}}$ and therefore $M \in \mathcal{C}_{\boldsymbol{f}}(q)$. Moreover, since $M \in S_{p} \mathcal{F}$, we have $|M: M \cap F|=p^{a}$, $\alpha>0$, and so by Doerk [2, Hilfssatz 1.2], $M^{p}$ has a nontrivial irreducible $Z_{q}[M]$-module $W$ such that $W_{F \cap M}$ contains the trivial $Z_{q}[F \cap M]$-module as a factor module. Since $F \cap M$ is an abnormal subgroup of $M$, certainly $\operatorname{dim}_{Z_{q}} W \neq 1$. Thus $W_{F \cap M}$ is not an irreducible $Z_{q}[F \cap M]$-module. Now, let $K=[W] M$, the semidirect product of $W$ by $M$. Since $F \cap M \in \mathcal{F}(q)$, it follows by Doerk [2, Hilfssatz 2.6], that $\bar{F}=[W](F \cap M)$ is an $\mathcal{F}$-projector of $K$. Also, since $M \in \mathcal{C}_{\mathcal{F}}(q), K \in \mathcal{C}_{\mathcal{F}}(q) \subseteq \mathcal{C}_{\mathcal{F}}$. However, $W$ is not a chief factor of $\bar{F}$, a contradiction. Hence, the lemma follows.

By an argument similar to the above, one can prove

Theorem 8. If $\mathfrak{X}$ is a saturated formation and $\Re \mathcal{F} \subseteq \mathcal{X} \subseteq \mathcal{C}_{\mathcal{F}}$, then $\pi_{\mathcal{F}}=$ X.

In view of Lemma 7 and Theorem 1.3 of Doerk and Hawkes [3] we have

Corollary 9. If $\mathcal{C}_{\mathcal{F}}$ is saturated; then $\mathcal{F}=S_{p}, \mathcal{F}(p)$ for some prime $p$.

We now prove Theorem 6.

Proof of Theorem 6. Suppose that $\mathcal{C}_{\boldsymbol{\mathcal { F }}}$ is saturated. In order to show that $\mathcal{F}=S$, it will be sufficient to show that $\mathcal{F}=\mathcal{F}(p)$ for each prime $p$. Suppose to the contrary that $\mathcal{F}=\mathcal{F}(p)$ for some prime $p$ and let $G \in \mathcal{F} \backslash \mathcal{F}(p)$ be of minimal order. Then $G$ contains a unique minimal normal subgroup $N$. Let $|N|=t^{\alpha}, t$ a prime and $\alpha>0$. Since $\mathcal{F}(p)=s_{p} \mathcal{F}(p), t \neq p$. 
Next, let $K$ be an isomorphic copy of the group $G$ in Huppert [9, Beispiel 2.9], if $p$ is odd; otherwise, let $K$ be the semidirect product of the natural 2-dimensional $\mathrm{GF}(5)$-module of $\mathrm{GL}(2,5)$ by the normalizer in $\operatorname{SL}(2,5)$ of a Sylow 2-subgroup of $\operatorname{SL}(2,5)$. In either case, $K$ has a unique minimal normal subgroup $M$ which is self-centralizing in $K$ and which is also a minimal normal subgroup of a Hall $p^{\prime}$-subgroup $K^{p}$ of $K$. Moreover, if $L / M=O_{p}(K / M)$, then $K / L$ is a $p^{\prime}$-group.

Consider now the wreath product $H=K\} G=\left[K_{1} \times \cdots \times K_{n}\right] G$ which is defined by a transitive permutation representation of $G$ of degree $n>1$. Since $K_{1} \times \cdots \times K_{n} / L_{1} \times \cdots \times L_{n}$ is a $p^{\prime}$-group and $G \in \mathcal{F}$, it follows from Corollary 9 that $H / L_{1} \times \cdots \times L_{n} \in \mathcal{F}$. Thus $H / M_{1} \times \cdots \times M_{n} \in \Re \mathcal{F} \subseteq \mathcal{C}_{\mathcal{F}}$. Also, since $M$ is an eccentric chief factor of $K^{p}$, it follows, by Hawkes [5, Lemma $]$, that $M_{1} \times \cdots \times M_{n}$ is a chief factor of $K^{p}\left\{G=\left[K_{1}^{p} \times \cdots \times K_{n}^{p}\right] G\right.$. But, by Carter and Hawkes [1, Theorem 5.12] applied to $H / M_{1} \times \cdots \times M_{n}$ and by another application of Corollary $9,\left[K_{1}^{p} \times \cdots \times K_{n}^{p}\right] G$ is a subgroup of some $\mathcal{F}$-projector $F$ of $H$. Hence, $F$ satisfies the hypothesis of Lemma 2, and so $H \in \mathcal{C}_{\mathcal{F}}$. However, $H \in \Re \mathcal{F}$, whereas $\Re \mathcal{F}=\mathcal{C}_{\mathcal{F}}$, by Lemma 7. Thus we have a contradiction. Hence, $\mathcal{F}=\mathcal{F}(p)$ for each prime $p$, and so $\mathcal{F}=S$. Since the converse of the theorem is trivially true, the proof is complete.

Acknowledgement. The authors are indebted to Dr. Trevor Hawkes for several very helpful conversations.

\section{REFERENCES}

1. R. W. Carter and T. O. Hawkes, The $\mathcal{F}$-normalizers of a finite soluble group, J. Algebra 5 (1967), 175-202. MR 34 \#5914.

2. K. Doerk, Zur Theorie der Formationen endlicher auflösbaren Gruppen, J. Algebra 13 (1969), 345-373. MR 40 \#237.

3. K. Doerk and T. Hawkes, Two questions in the theory of formations, J. Algebra 16 (1970), 456-460. MR 42 \#7775.

4. W. Gaschütz, Zur Theorie der endlichen auflösbaren Gruppen, Math. Z. 80 (1962/63), 300-305. MR 31 \#3505.

5. T. O. Hawkes, An example in the theory of soluble groups, Proc. Cambridge Philos. Soc 67 (1970), 13-16. MR 40 \# 1477.

6. B. Huppert, Endliche Gruppen I, Die Grundlehren der math. Wissenschaften, Band 134, Springer-Verlag, Berlin and New York, 1967. MR 37 \#302.

7. — Zur Theorie der Formationen, Arch. Math. (Basel) 19 (1968), 561574 (1969). $\quad$ MR $39 \# 5697$.

DEP ARTMENT OF MATHEMATICS, UNIVERSITY OF KENTUCKY, LEXINGTON, KENTUCKY 40506 